\title{
The IAU and hazardous Near Earth Objects - a clear and present danger
}

\author{
Karel A. van der Hucht
}

IAU General Secretary 2006-2009

SRON Netherlands Institute for Space Research, NL-3584 CA Utrecht, the Netherlands email: k.a.van.der.hucht@sron.nl

\begin{abstract}
The Minor Planet Center, established in 1947 by the IAU, is the international repository and clearinghouse for the world's minor planet observations. Since 1989, CCD surveys of Near Earth Objects at ground-based astronomical observatories are operational, mainly in the USA. As of 23 August, 2018, a total of 18,545 Near Earth Asteroids (NEAs) and 107 Near Earth Comets (NECs) have been registered and daily updates are made publicly available on the internet by the MPC, NASA-JPL-CNEOS and ESA-SSA-NEOCC.

Concern about the possibility of NEO impacts has been picked up by the United Nations Committee on the Peaceful Uses of Outer Space (UN-COPUOS), where the IAU has observer status, and formally expressed since 1999. This led in 2014 to the formation of two international coordinating bodies for NEO detection and NEO impact mitigation: the International Asteroid Warning Network (IAWN) and the Space Mission Planning Advisory Group (SMPAG).

In support of these developments, the IAU 28th General Assembly, Session II, held in Beijing on 30 August 2012, adopted a Resolution (3B) recommending the establishment of an International NEO Early Warning System, as proposed by the IAU Division III (now Division F) Working Group on Near-Earth Objects. The GA recommended "...that the IAU National Members work with the United Nations Committee on the Peaceful Uses of Outer Space (UNCOPUOS) and the International Council for Science (ICSU), to coordinate and collaborate on the establishment of an International NEO Early Warning System, relying on the scientific and technical advice of the relevant astronomical community, whose main purpose is the reliable identification of potential NEO collisions with the Earth, and the communication of the relevant parameters to suitable decision makers of the nation(s) involved. ...."
\end{abstract}

The NEO hazard issue received world-wide attention on 13 February 2013 when a NEA with an estimated size of 17 to 20 meters and an estimated mass of 11,000 tons exploded over Chelyabinsk (Russia), releasing $440 \mathrm{kT}$ TNT of energy at an altitude of $\sim 23 \mathrm{~km}$.

Subsequently, on 5 December 2014, the United Nations General Assembly adopted a Resolution (69/85, 9-10), noting "... the importance of information-sharing in discovering, monitoring and physically characterizing potentially hazardous near-Earth objects to ensure that all countries, in particular developing countries with limited capacity in predicting and mitigating a near-Earth object impact, are aware of potential threats, emphasizes the need for capacitybuilding for effective emergency response and disaster management in the event of a near-Earth object impact, ...."

In spite of all dedicated NEO surveys operational to date, the present inventory and thus our assessment of the level of threat of NEOs is severely limited by their huge number and by the available observational capabilities. E.g., while the estimated number of all NEOs larger than 40 meters in diameter is $\sim 700,000$, only $\sim 2 \%$ have been detected to date. For NEOs with sizes between 40 and 140 meters, the detection percentage amounts to less than $1 \%$ of the estimated number. Only dedicated space-based surveys, preferably in the infrared, will be able to provide the much needed orders of magnitude improvement in the detection, tracking and characterizing of NEOs. One promising project is the NASA-JPL NEOCam mission, studied since 2005 but not yet approved: a dedicated infrared observatory aiming to detect, track and characterize NEO's from the Sun-Earth Lagrange point L1. 
As Yeomans puts it: we better find them before they find us (Yeomans 2013). Traditionally, astronomers are looking back into the past, if only because of the limited speed of light. But we should realize that the clear and present danger posed by hazardous Near Earth Objects to mankind and all other life forms obliges us to look also forward, into the future. Provided with the proper means, we astronomers can do that, as a small service to society, including ourselves. The astronomical community at large should give high priority to NEO survey projects, in particular space-based surveys.

Keywords. Near Earth Objects, Near Earth Asteroids, Near Earth Comets, Potentially Hazardous Objects, NEO Early Warning System

\section{Introduction}

In 1980, the relevance of the cosmic environment to the nature of life and evolution received a stunning boost from the discovery that collisions of asteroids and comets with the Earth have affected terrestrial evolution in dramatic ways in the past, and thus that they will do so again in the future (Alvarez 1980a,b; Hildebrand 1991; Hand 2016). Since then, the astronomical discipline of discovering, tracking and characterizing asteroids and comets has matured.

An object is coined a Near Earth Object (NEO) when its trajectory brings it within 1.3 AU from the Sun. A NEO is said to be a Potentially Hazardous Object (PHO) when its orbit comes to within 0.05 AU of the Earth's orbit and has an estimated diameter $D>140 \mathrm{~m}$ (Yeomans 2013). In January 1980, the orbits of only 51 Near Earth Asteroids (NEAs) and 44 Near Earth Comets (NECs), together named Near Earth Objects (NEOs), were known $\dagger$. As of 23 August 2018, orbits of 18,545 NEAs and 107 NECs are publicly available on the internet, thanks to the cumulative efforts of the Minor Planet Center (MPC) $\ddagger$, the NASA JPL Center for NEO Studies (CNEOS) $\uparrow$, and the ESA SSA NEO Coordination Center (NEOCC)\|. Because the number of NECs is less than $1 \%$ of that of NEAst†, we focus on NEAs in the further discussion.

Since A.D. 1900, 433 past close approaches of NEAs with nominal Earth close approach distances $d=1.00 \mathrm{LD}$ are registered, ranging in size from 1 to $325 \mathrm{~m}$ (54 of those occurred in 2017, and 40, to date, in 2018), 23 of which with $d=0.10$ LD. Five impacts have been recorded: in 1908, 2008, 2013, 2014, and 2018. Moreover, the available orbit calculations for known NEAs predict only 36 future close approaches of NEAs with nominal Earth close approach distances $d=1.00$ LD till A.D. 2200 + . A factor of 1000 short. Calculations until that date show that none of the presently known NEAs $₫$ will impact with the Earth. But what about the unknown NEAs?

What degree of completeness has been achieved to date for this inventory? Our present knowledge, notably of NEAs less than $140 \mathrm{~m}$ in size, is severely limited (Harris 2017a,b). In recent years, all the world's ground-based surveys together, operating primarily in the USA, discovered some 2000 new NEAs per year \|\| . At the present discovery rate, expanding the inventory to encompass, say, 90\% of all NEAs larger than 40 meters,

\footnotetext{
$\dagger$ cneos.jpl.nasa.gov/stats/totals.html

$\ddagger$ www.cfa.harvard.edu/iau/mpc.html

II cneos.jpl.nasa.gov/stats/totals.html

|| neo.ssa.esa.int

$\dagger \dagger$ cneos.jpl.nasa.gov/stats/totals.html

$\ddagger \ddagger$ cneos.jpl.nasa.gov/stats/totals.html

ๆ ๆttps://ssd.jpl.nasa.gov/sbdb.cgi

|| || cneos.jpl.nasa.gov/stats/totals.html
} 
would take many centuries. The impact danger of NEAs is not so much associated with those presently known, but with the NEAs which have not yet been discovered.

\section{Why all those efforts, why bother? A brief history}

Numerous large NEO impacts have occurred in prehistoric times. The Earth Impact Database lists 160 confirmed impact sites with crater diameters in the range 10-300 $\mathrm{km} . \dagger$ The recorded number of relatively small NEO impacts in the previous and present century shows that our place in the Solar System is not so safe as we may have thought, say, 50 years ago. We present here a small selection of relevant impact events.

66 Myr BC: Chicxulub crater (Mexico). The $\sim 150 \mathrm{~km}$ wide and $\sim 20 \mathrm{~km}$ deep Chicxulub impact crater, buried beneath the Yucatn Peninsula (Mexico), was formed by the impact of a large asteroid or comet about 10 to $15 \mathrm{~km}$ in diameter. The time of the impact coincides precisely with the CretaceousTertiary boundary (KT boundary), slightly less than 66 million years ago. The impact caused a worldwide climate disruption leading to a mass extinction in which $\sim 75 \%$ of all plant and animal species on Earth disappeared, including all non-avian dinosaurs. In its orbit, the Earth moves a distance equal to its own diameter in $\sim 7$ minutes. Thus, if the asteroid or comet that triggered this extinction had arrived 7 minutes earlier or later, it would have missed our planet, the dinosaurs might have prevailed and we would not be here now. The comet or asteroid impact of 66 million years ago was not the first of its kind, nor will it be the last. Terrestrial life - of all kinds - remains vulnerable to devastating blows from space, at least until such time as we decide to do something to protect our planet from a potentially lethal impact. And from less-than-lethal impacts, for that matter (Alvarez 1980a,b; Hildebrand 1991; Hand 2016).

50000 B.C.: Meteor Crater (near Winslow, AZ, USA). Meteor Crater, thought to have been created by the impact of a 40-50 m-sized iron asteroid about 50,000 years ago, is $1.2 \mathrm{~km}$ in diameter and $170 \mathrm{~m}$ deep. No iron mass has been found below the crater floor, but numerous iron meteorites have been discovered in the region surrounding the crater (Hand 2016; Shoemaker 1963; Roddy and Shoemaker 1995).

1490: Meteorite fall (China). In February-March of the year 1490 a barrage of stones weighing 1-1.5 kg fell like rain in the Ching-yang district of Shansi Province (China), killing more than 10,000 people (Yau et al. 1994).

1803: L'Aigle Meteorite Fall (France). L'Aigle Meteorite Fall. In the early afternoon of 26 April 1803 a meteorite shower of more than 3000 fragments fell upon the town of L'Aigle (Normandy, France) (Gounelle 2003).

1908: Tunguska (Russia), 30 June 1908. On 30 June 1908, an asteroid with $D \approx 40 \mathrm{~m}$ exploded in an airburst with an energy release of about 4 megaton TNT at an altitude of $\sim 8.5 \mathrm{~km}$. This flattened some $2000 \mathrm{~km}^{2}$ of forest near the Tunguska river (Central Siberia, Russia) (Boslough and Crawford 2008; Napier and Asher 2009).

1994: Comet Shoemaker-Levy 9, impacting on Jupiter. Comet Shoemaker-Levy 9, originally $\sim 10 \mathrm{~km}$ across, was discovered in a two-year orbit around Jupiter. In July 1992 the comet had come so close to Jupiter that it had been broken up by the planet's gravitational pull into 21 pieces, each about 1-4 km across, impacting on Jupiter on 17-22 July 1994 (Levy et al. 1995; Weaver et al. 1995).

2007: Carancas (Peru). A 1 m-sized asteroid is given credit for creating a nearly 4-m diameter crater near the village of Carancas (Peru) near Lake Titicaca and the Bolivian border, on 15 September 2007. The explosion was heard some $20 \mathrm{~km}$ from the impact site and caused broken window glass about $1 \mathrm{~km}$ from ground zero (Borovicka and Spurný 2008).

2008: NEA 2008 TC3 down in Sudan. Asteroid 2008 TC3 ( $D \approx 4.1 \mathrm{~m}$ ) was discovered only 19 hours before disintegrating in a fireball and airburst over the Nubian desert

$\dagger$ www.passc.net/EarthImpactDatabase 
Table 1. Average impact results by size, from Yeomans (Yeomans 2013, p. 115).

\begin{tabular}{lllll}
\hline $\begin{array}{l}\text { diameter of } \\
\text { impactor }\end{array}$ & $\begin{array}{l}\text { total NEA } \\
\text { population }\end{array}$ & $\begin{array}{l}\text { typical } \\
\text { impact energy }\end{array}$ & $\begin{array}{l}\text { average interval } \\
\text { between impacts }\end{array}$ & $\begin{array}{l}\text { crater } \\
\text { diameter }\end{array}$ \\
\hline $1 \mathrm{~m}$ & $\sim 1$ billion & $47 \mathrm{~T}$ & 2 weeks & no crater \\
$10 \mathrm{~m}$ & $\sim 10 \mathrm{million}$ & $47 \mathrm{KT}$ & $10 \mathrm{yr}$ & no crater \\
$30 \mathrm{~m}$ & $\sim 1.3 \mathrm{million}$ & $1.3 \mathrm{MT}$ & $200 \mathrm{yr}$ & no crater \\
$100 \mathrm{~m}$ & $\sim 28,000$ & $47 \mathrm{MT}$ & $5,200 \mathrm{yr}$ & $1.2 \mathrm{~km}$ \\
$140 \mathrm{~m}$ & $\sim 16,000$ & $129 \mathrm{MT}$ & $13,000 \mathrm{yr}$ & $2.2 \mathrm{~km}$ \\
$500 \mathrm{~m}$ & $\sim 28,000$ & $5,870 \mathrm{MT}$ & $130,000 \mathrm{yr}$ & $7.4 \mathrm{~km}$ \\
$1,000 \mathrm{~m}$ & $\sim 921$ & $47,000 \mathrm{MT}$ & $440,000 \mathrm{yr}$ & $13.6 \mathrm{~km}$ \\
$10,000 \mathrm{~m}$ & 4 & $47,000 \mathrm{GT}$ & $89,000,000 \mathrm{yr}$ & $104 \mathrm{~km}$ \\
\hline
\end{tabular}

in northern Sudan. Immediately after discovery, the international observers' community responded with some 570 timely observations from 27 observers of that NEA. A systematic search in the desert located 280 meteorite fragments with a total mass of $3.9 \mathrm{~kg}$ (Jenniskens 2009).

2013: Chelyabinsk (Russia). The NEO hazard issue received world-wide attention when on 13 February 2013 a NEA with an estimated size of 17-20 m and an estimated mass of 11,000 tons exploded over Chelyabinsk (Russia), releasing $440 \mathrm{kT}$ TNT of energy at an altitude of $\sim 23 \mathrm{~km}$. The airburst shock wave, arriving 88 seconds after the superbolide's flash, damaged numerous buildings and blew out thousands of glass windows. Over 1500 people needed medical attention for minor injuries. Three meteorite debris impact sites were located. The asteroid was an estimated 24th mag object just before impact, when it approached the Earth within 15 degrees of the direction of the Sun. The event took 32.5 seconds from atmospheric entry of the NEA, with a velocity of $\sim 18.6 \mathrm{~km} / \mathrm{s}$, to the its airborne disintegration (Borovicka 2013; Brown 2013; Popova 2013).

\section{What are the risks?}

The 2017 Report of the NASA NEO Science Definition Team states:

"We estimate a population of about 934 NEOs larger than 1 kilometer, leading to an impact frequency of about one in a half a million years. The population of NEOs with diameters of 140 meters and larger is estimated to be $\sim 25,000$. To the lower limit of an object's atmospheric penetration (about 50 meters in diameter for non-metallic objects), we estimate about half a million NEOs, with an impact frequency of about one in a thousand years." (Stokes 2017)

While the impact frequencies of these large NEOs are small, the consequences of such impacts can be seriously dramatic. Yeomans lists rough estimates of the population of stony NEAs of various sizes and their average impact effects (Yeomans 2013) (see Table 1).

The short-term risk is for NEAs with sizes in the range 20 to 40 meters, as demonstrated by the 1908 Tunguska and 2013 Chelyabinsk impact events.

A Tunguska impactor-sized object passes within the lunar distance from Earth several times per year. A Tunguska impactor-sized asteroid will strike Earth every 500 years. An asteroid the size of the Chelyabinsk impactor passes within geosynchronous orbit every two years, and within the Moon's orbit nearly once a week. Roughly 10 million Chelyabinsk-sized objects have Earth crossing orbits, and the impact interval is closer to 50 years (Eicher 2015).

\section{International activities and developments}

A previous report on the involvement of the IAU on the subject of Near Earth Objects was presented by van der Hucht \& Andersen (van der Hucht and Andersen 2015). 
The Minor Planet Center. Established in 1947, the IAU Minor Planet Center (MPC) was hosted initially by the Observatory of the University of Cincinnati (OH, USA), and since 1978 by the Smithsonian Astrophysical Observatory in Cambridge (MA, USA) †, (Bardwell 1989; Marsden 2009). The MPC has developed into the vast international repository and clearinghouse for the world's minor planet observations, including NEOs, i.e., NEAs and NECs, with an extensive range of online data and orbit computing servicesł.

Initially, the MPC operated on a series of informal agreements with the International Astronomical Union, with no written contract or equivalent. By the year 2000, the magnitude of its operations and public awareness of the NEO impact hazard had developed to a level where the MPC became equal in visibility and in financial terms to the whole of the IAU. Formal Terms of Reference for the MPC were therefore agreed with its host and sponsors, to ensure the continued viability of the MPC and free access for all qualified researchers to the holdings of its data bank.

For decades observatories and observers (professionals and amateurs) from around the world have been sending NEO astrometric observations, from which orbits can be calculated, to the MPC. The MPC collects these data, verifies them, provides object designations and discovery credits, and makes the data publicly available, notably to the NASA Center for NEO Earth Object Studies (CNEOS) at the NASA Jet Propulsion Laboratory (JPL) in La Cañada (CA, USA) $\uparrow$, the ESA NEO Coordination Center (NEOCC) at the ESA European Space Research Institute (ESRIN) in Frascati (Italy)\|, and the ESA Near-Earth Object Dynamic Site (NEODyS-2) which has offices in Pisa, Rome and Cascina (Italy) $\dagger \dagger$.

The IAU takes pride in its association with the MPC, which operates under the auspices of IAU Division F (formerly Division III) and, before that, Commission 20, while currently deriving its operating budget from a five-year NASA grant. Its present staff includes Matthew J. Holman, director, and Gareth V. Williams, associate director. Since June 2018, the MPC is also operating under the NASA Planetary Data Systems Small Bodies Node (SBN). The SBN serves as intermediary between the direction of the NASA Planetary Defense Coordination Office and the MPC $\neq \ddagger$.

The United Nations Committee on the Peaceful Uses of Outer Space (UN-COPUOS). Since 1999, concern about NEO impacts has been expressed by the United Nations Committee on the Peaceful Uses of Outer Space (UN-COPUOS), where the IAU has observer status $₫$. Notably the UN-COPUOS Scientific and Technical Subcommittee (STsC) has NEOs explicitly on its agenda \|\| . The STsC was advised by UNISPACE III Action Team 14 on NEOs, with the following Terms of Reference (ToR):

(a) review the content, structure and organization of on-going efforts in the field of NEOs;

(b) identify any gaps in the on-going work where additional cooperation is required and/or where other countries or organizations could make contributions; and

(c) propose steps for the improvement of international coordination in collaboration with specialized bodies.

$\dagger$ www.minorplanetcenter.net/iau/mpc.html

$\ddagger$ www.cfa.harvard.edu/iau/mpc.html

I cneos.jpl.nasa.gov/stats/totals.html

|| neo.ssa.esa.int

$\dagger \dagger$ newton.dm.unipi.it/neodys/index.php?pc=0

$\ddagger \ddagger$ www.lpi.usra.edu/sbag/meetings/jun2018/presentations/fast.pdf

ๆ 『 ww.unoosa.org/oosa/en/ourwork/copuos/index.html

|| || www.unoosa.org/oosa/en/ourwork/copuos/stsc/index.html 
The ToR for Action Team 14 were formulated in July 1999 in Vienna (Austria) at the Third United Nations Conference on the Exploration and Peaceful Uses of Outer Space: UNISPACE III $\dagger$. From 2003 to 2012 the IAU was represented in Action Team 14 by K.A. van der Hucht (IAU General Secretary 2006-2009) (van der Hucht and Andersen 2015). A decisive role in Action 14 was played by the Association of Space Explorers Committee on Near-Earth Objects through its Panel on Asteroid Threat Mitigation, chaired by R.L. Schweickart, and providing the report Asteroid Threats: a Call for Global Response in September 2008 .

The International Astronomical Union 9 . In support of these efforts, the IAU 28th General Assembly, Session II, held on 30 August 2012 in Beijing, adopted its Resolution 3B, recommending establishment of an International NEO Early Warning System, as proposed by the IAU Division III (now Division F) Working Group on Near-Earth Objects $\|$. The Resolution reads (Montmerle 2015):

The XXVIIIth General Assembly of the International Astronomical Union, recognizing

- that there is now ample evidence that the probability of catastrophic impacts of Near-Earth Objects (NEOs) onto the Earth, potentially highly destructive to life, and for humankind in particular, is not negligible and that appropriate actions are being developed to avoid such catastrophes;

- that for the largest NEOs, thanks to the efforts of the astronomical community and of several space agencies, the cataloguing of the potentially hazardous ones, the monitoring of their impact possibilities, and the analysis of technologically feasible mitigations is reaching a satisfactory level;

- that even the impact of small- to moderate-sized objects may represent a great threat to our civilizations and to the international community;

- that our knowledge of the number, size, and orbital behavior of smaller objects is still very limited, thus not allowing any reasonable anticipation on the likelihood of future impacts,

noting

that NEOs are a threat to all nations on Earth, and therefore that all nations should contribute to avert this threat,

recommends

that the IAU National Members work with the United Nations Committee on the Peaceful Uses of Outer Space (UN-COPUOS) and the International Council for Science (ICSU) to coordinate and collaborate on the establishment of an International NEO Early Warning System, relying on the scientific and technical advice of the relevant astronomical community, whose main purpose is the reliable identification of potential NEO collisions with the Earth, and the communication of the relevant parameters to suitable decision makers of the nation(s) involved.

The activities of the IAU Division F Working Group on Near Earth Objects have expanded considerably in the meantime (Michel 2018).

The United Nations General Assembly. The recommendations of the UN-COPUOS Scientific Technical Subcommittee Action Team 14 on NEOs to the United Nations General Assembly resulted in Resolution 69/85 adopted by the UN General Assembly on 5 December $2014 \uparrow \dagger$ :

$\dagger$ www.oosa.unvienna.org/oosa/unisp-3/index.html $\ddagger$ www.space-explorers.org/committees/NEO/docs/ATACGR.pdf

बा www.iau.org

|| www.iau.org/science/scientific_bodies/working_groups/171

$\dagger \dagger$ www.unoosa.org/pdf/gares/A_RES_69_085E.pdf 
"The General Assembly,...

9. Notes the importance of information-sharing in discovering, monitoring and physically characterizing potentially hazardous near-Earth objects to ensure that all countries, in particular developing countries with limited capacity in predicting and mitigating a near-Earth object impact, are aware of potential threats, emphasizes the need for capacitybuilding for effective emergency response and disaster management in the event of a near-Earth object impact, and recalls in that regard the recommendations for an international response to the near-Earth object impact threat, endorsed by the Scientific and Technical Subcommittee at its fiftieth session and by the Committee [UN-COPUOS] at its fifty-sixth session;

10. Notes with satisfaction that progress on establishing an international asteroid warning network and a space mission planning advisory group to implement the recommendations for an international response to the near-Earth object impact threat would be reported to the Subcommittee at its fifty-second session."

Thus, the United Nation's General Assembly endorsed the final report of Action Team 14 of UN-COPUOS that recommended for the Science Technical Sub-committee the specific task of coordinating the international response to NEO impact threats. This included the establishment of an International Asteroid Warning Network (IAWN) and of a Space Mission Planning Advisory Group (SMPAG).

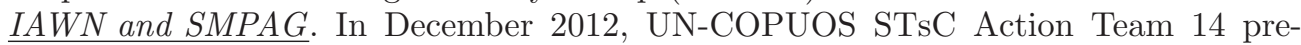
sented its Final Report to the UN-COPUOS STsC $\dagger$. Its recommendation for the establishment of the above-mentioned two new internationally coordinating bodies for NEO detection and NEO impact mitigation: the International Asteroid Warning Network (IAWN) and the Space Mission Planning Advisory Group (SMPAG), was approved by the STsC and came into effect in 2014 $\ddagger$. The UN-COPUOS STsC noted in its report of February 2015 that Action Team 14 on Near-Earth Objects had successfully established IAWN and SMPAG, and recommended that Action Team 14 be dissolved $\boldsymbol{\uparrow}$.

The International Asteroid Warning Network (IAWN). IAWN was established to create an international group of organizations involved in detecting, tracking and characterizing NEOs $\|$. IAWN is tasked with developing a strategy using well-defined communication plans and protocols to assist governments in the analysis of asteroid impact consequences and in the planning of mitigation responses. IAWN's functions are:

- to discover, monitor, and physically characterize the potentially hazardous NEO population using optical and radar facilities and other assets based in both the northern and southern hemispheres and in space;

- to provide and maintain an internationally recognized clearinghouse function for the receipt, acknowledgement and processing of all NEO observations;

- to act as a global portal, serving as the international focal point for accurate and validated information on the NEO population;

- to coordinate campaigns for the observation of potentially hazardous objects;

- to recommend policies regarding criteria and thresholds for notification of an emerging impact threat;

$\dagger$ Near-Earth objects, 2012-2013, Final report of the Action Team on Near-Earth Objects, www.unoosa.org/pdf/limited/c1/AC105_C1_L330E.pdf

$\ddagger$ Report of the Scientific and Technical Subcommittee on its fifty-first session, Vienna, 10-21 February 2014, www.unoosa.org/pdf/reports/ac105/AC105_1065E.pdf; Report of the Committee on the Peaceful Uses of Outer Space Fifty-eighth Session, Vienna, 10-19 June 2015, www.unoosa.org/res/oosadoc/data/documents/2015/a/a7020_0_html/A_70_20E.pdf

I Report of the Scientific and Technical Subcommittee on its fifty-second session, Vienna, 2-13 February 2015, www.unoosa.org/pdf/reports/ac105/AC105_1088E.pdf, (184-188)

|| iawn.net 
- to develop a database of potential impact consequences, depending on geography, geology, population distribution and other related factors;

- to assess hazard analysis results and communicate them to entities that should be identified by Member States as being responsible for the receipt of notification of an impact threat in accordance with established policies;

- to assist Governments in the analysis of impact consequences and in the planning of mitigation responses.

The current signatories of the IAWN Statement of Intent includet: CNSA (Chinese National Space Administration), CrAO (Crimean Astrophysical Observatory, Russian Academy of Sciences), ESA (European Space Agency), ESO (European Southern Observatory), INAOE (National Institute of Astrophysics, Optics, and Electronics in Cholua, Mexico), INASAN (the Institute of Astronomy, Russian Academy of Sciences), ISTP (Institute of Solar-Terrestrial Physics, Russian Academy of Sciences), KAO UrFU (Kourovka Astronomical Observatory of the Ural Federal University), KASI (Korea Astronomy Space Science Institute, Daejeon, South Korea), SAO RAS (Special Astrophysical Observatory of the Russian Academy of Sciences), NASA (National Aeronautics and Space Administration, United States), and University of Narino, Pasto, Colombia.

IAWN reports to the UN-COPUOS Scientific and Technical Subcommitteef.

The Space Mission Planning Advisory Group (SMPAG). The primary objective of the SMPAG is to prepare for an international response to a NEO threat through the exchange of information and development of options for collaborative research and missions, and to conduct planning activities for NEO threat mitigation $\boldsymbol{~}$. Its Terms of Reference are listed in $\|$.

Present membership of SMPAG comprises the space agencies: AEM (Mexico), ASI (Italy), BELSPO (Belgium), CNSA (China), CNES (France), DLR (Germany), ESA, FFG-ARPA (Austria), IAWN (ex-officio), ISA (Israel), JAXA (Japan), KASI (Korea), NASA (USA), SUPARCO (Pakistan), ROSA (Romania), ROSCOSMOS (Russian Federation), SSAU (Ukraine), and UK Space Agency (UK).

Observer status is held by: ASE (Association of Space Explorers), ESO (European Southern Observatory), IAA (International Academy of Astronautics), IAU (International Astronomical Union), and the UN Office of Outer Space Affairs (OOSA).

SMPAG reports to the U N-COPUOS Scientific and Technical Subcommitteet†.

Asteroid Day. Asteroid Day (Eicher 2015) $\ddagger \ddagger$, also known as International Asteroid Day, is a United Nations sanctioned annual global awareness event, to be held on the anniversary of the Siberian Tunguska asteroid impact of 30 June 1908, the most harmful known asteroid-related event on Earth in recent history (Boslough and Crawford 2008; Napier and Asher 2009). Asteroid Day was co-founded in 2015 by filmmaker Grigorij Richters, B612 Foundation COO Danica Remy, Apollo-9 astronaut Rusty Schweickart, and Queen guitarist and astrophysicist Brian May. The proposal for Asteroid Day was processed through the UN-COPUOS Scientific and Technical Subcommittee and approved by the United Nations General Assembly 71st session on 6 December 2016 $\mathbf{\Upsilon}$.

In its Resolution the United Nations General Assembly declares |||:

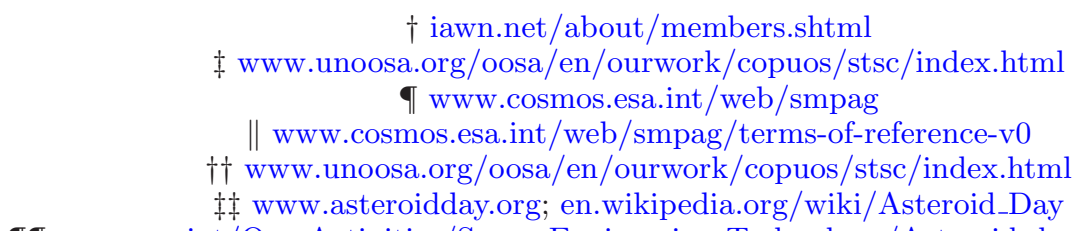

ๆ ฯ www.esa.int/Our_Activities/Space_Engineering_Technology/Asteroid_day/UN_recognises_ 30_June_as_Asteroid_Day

||| www.un.org/en/ga/71/resolutions.shtml 
“. . 30 June International Asteroid Day to observe each year at the international level the anniversary of the Tunguska impact over Siberia, Russian Federation, on 30 June 1908 and to raise public awareness about the asteroid impact hazard."

\section{An operational space-based infrared NEO survey: NEOWISE}

The NEOWISE project focusses on asteroid-hunting as part of the NASA Wide-field Infrared Survey Explorer (WISE) mission. Launched in December 2009, WISE surveyed the full sky in four infrared wavelength bands $(3.4,4.6,12,22 \mu \mathrm{m})$ till September 2010. The survey continued as NEOWISE for an additional four months using the two shortest wavelength detectors. The spacecraft was put into hibernation in February 2011, after completing its search of the inner solar system. During its primary mission, NEOWISE delivered infrared detections of more than 158,000 minor planets to the MPC, including more than 34,000 new discoveries. NEOWISE was brought out of hibernation in December 2013 to detect NEOs in the 3.4 and $4.6 \mu \mathrm{m}$ infrared wavelength bands. In midJuly 2018, NEOWISE was $25 \%$ through its tenth coverage of the sky since the start of the reactivation mission. Over 689,000 infrared measurements have been made by NEOWISE of 33,328 different solar system objects, including 875 NEOs and 148 comets $\dagger$.

\section{Future surveys}

In spite of all the dedicated ground-based surveys and the data amassed from the ongoing space-based NEOWISE survey, the sheer number of NEOs and their presently known number are seriously out of balance; appreciation of the level of threat from NEOs is severely limited by the available observational capabilities. Is the astronomical community striving to improve this dire situation? Certainly, although funding is still far from sufficient. We refer here to one funded future ground-based survey telescope project, LSST, and to one funded study for a future space-based infrared survey telescope project, NEOCam, still awaiting its final approval.

The Large Synoptic Survey Telescope. The Large Synoptic Survey Telescope (LSST) is a 6.4-m equivalent ground-based telescope with first-light foreseen in $2022 \ddagger$. It will devote part of its telescope time to the search for NEOs. In between its other tasks and depending on its mode of operation, LSST should be able to discover about $60 \%$ of the potentially hazardous NEAs larger than $140 \mathrm{~m}$ (PHAs) in the course of its projected 10-yr survey lifetime (Grav et al. 2016; Vereš and Chesley 2017).

NEOCam. Obviously, only dedicated continuously operational space-based surveys will be able to provide improvement in discovery, tracking and characterizing NEOs by the requisite orders of magnitude. Since 2005, one space-based NEO survey mission is being studied: the NASA-JPL NEOCam project, currently in Extended Phase A. It is conceived around a 50-cm telescope with a wide-field infrared camera, to observe at thermal infrared wavelengths of 4-10 m, with detectors passively cooled down to $40 \mathrm{~K}$. NEOCam aims to operate in the Sun-Earth Lagrange point L1 for at least five years and should discover in that lifetime two-thirds of the estimated number of NEAs larger than $140 \mathrm{~m} \uparrow$.

\section{Concluding remarks}

NEO impacts are unique among natural hazards in that they can be predicted with great precision and can (in principle) be avoided by application of advanced space technology. For the first time in our planet's history, the technical capacities exist to prepare for NEO collisions with Earth. But only in a sensible way, when a more or less complete

$\dagger$ neowise.ipac.caltech.edu

$\ddagger$ www.lsst.org/lsst; b612foundation.org/facing-the-future-of-lsst-era-hazardous-asteroiddiscovery

\ neocam.ipac.calteh.edu 
inventory of the NEO populations is achieved. International cooperation in NEO data collection is mandatory for this endeavor. As Yeomans puts it:

"Near Earth Objects are among the smallest members of the solar system, but their diminutive size is in no way proportional to their importance. ... We better find them, before they find us." (Yeomans 2013)

As the international organization uniting the world's astronomical community, the IAU should assert its influence on all astronomical institutes and organizations of the world and its sponsors to support, in one way or the other, the realization and permanent operation of a series of space-based NEO survey observatories, in order to boost the inventory to a level of at least $90 \%$ of all NEOs larger than $40 \mathrm{~m}$ within a reasonable time. Only then could it be possible to issue timely warnings of possible NEO impacts of the Tunguska impact level and worse to endangered parts of the world's population, so that evacuation and mitigation plans can be prepared to cope with the threat. Regardless of the costs.

As a logical consequence of IAU Resolution 3B of the IAU 28th General Assembly in 2012, recommending the establishment of an International NEO Early Warning System $\dagger$, it would be wise if the world's astronomical community would decide to earmark a reasonable percentage, say, $2 \%$, of its annual budget to the astronomical aspects of planetary defense. As an insurance policy for planet Earth against NEO impacts, those loose cannons of the cosmos.

The words of the late Harvard Observatory director Fred L. Whipple (1985) remain valid:

"Protection of the Earth from undesirable impacting bodies is not just a science fiction project for some improbable future. The cost might be comparable to, even smaller than, the worlds current military expenditures. We could choose to do it now. We could choose to protect ourselves from asteroids and comets, rather than from each other." (Whipple 1985).

\section{Acknowledgements}

Critical reading of the manuscript by Mme Vivien Reuter is gratefully acknowledged.

\section{References}

Alvarez, L. W., Alvarez, W., and Klint, S. 1980a, Science News, 117, 22

Alvarez, L. W., Alvarez, W., Asaro, F., and Michel, H. V. 1980b, Science, 208, 1095

Bardwell, C. M. 1989, Temmon Guide, www.oaa.gr.jp/oaacs/mp/BriefHistoryofMPCbyConrad Bardwell.pdf

Borovicka, J. and Spurný, P. 2008, Astronomy and Astrophysics, 485, 1

Borovicka, J., Spurný, P., Brown, P., et al. 2013, Nature, 503, 235

Boslough, M. B. E. and Crawford, D. A. 2008, Intern. J. of Impact Engineering, 35, 1441

Brown, P. G., Assink, J. D., Astiz, L., et al. 2013, Nature, 503, 238

Eicher, D. J. 2015, Why the asteroid threat should be taken seriously, Astronomy Magazine, 24 June 2015

Gounelle, M. 2003, in: 66th Annual Meteoritical Society Meeting, Meteoritics and Planetary Science, 38, Supplement, abstract no.5251

Grav, T., Mainzer, A., and Spahr, T. 2016, Astronomical Journal, 151, 172

Hand, E. 2016, Science News, 17 November 2016

Harris, A. W. 2017a, AAS DPS meeting 49, id.\#100.01

Harris, A. W. 2017b, More Data!, Phys.Org News, 19 October 2017

Hildebrand, A. R., Penfield, G. T., Kring, D. A., et al. 1991, Geology, 19, 867

$\dagger$ www.iau.org/science/scientific_bodies/working_groups/171 
Jenniskens, P., Shaddad, M. H., Numan, D., et al. 2009, Nature, 458, 485

Levy, D. H., Shoemaker, E. M., and Shoemaker, C. S. 1995, Scientific American, 273, 68

Marsden, B. G. 2009, IAU Information Bulletin, No.104, p. 67, www.iau.org/static/ publications/IB104.pdf

Michel, P. 2018, Report of the IAU Near Earth Object Working Group 20152018, www.iau.org/static/science/scientific_bodies/working_groups/171/wg-neos-triennialreport-2015-2018.pdf

Montmerle, T. (ed.) 2015, Transactions IAU XXVIIIB, Proceedings of the Twenty Eighth General Assembly, Beijing, China, 2012, p.39

Napier, B. and Asher, D. 2009, Astronomy and Geophysics, 50, 1.18

Popova, O. P., Jenniskens, P., Emelýanenko, V., et al. 2013, Science, 342, 1069

Roddy, D. J. and Shoemaker, E. M. 1995, Meteoritics, 30, 567

Shoemaker, E. M. 1963, in: B. M. Middlehurst and G. P. Kuiper (eds.), The Moon, Meteorites, and Comets, (Chicago: UCP), p. 301

Stokes, G. H., Barbee, B. W., Bottke, W. F., et al. 2017, Report of the [NASA] Near-Earth Object Science Definition Team, 2017

van der Hucht, K. A. and Andersen, J. 2015, in: J. N. Pelton and F. Allahdadi (eds.), Handbook of Cosmic Hazards and Planetary Defense (Heidelberg: Springer), p.755

Vereš, P. and Chesley, S. R. 2017, Astronomical Journal, 154, 13

Weaver, H. A., A'Hearn, M. F., Arpigny, C., et al. 1995, Science, 267, 1282

Whipple, F. L. 1985, The Mystery of Comets, (Baltimore: Smithsonian Institution Press).

Yau, K., Weissman, P., and Yeomans, D. K. 1994, Meteoritics, 29, 864

Yeomans, D. K. 2013, Near-Earth Objects. Finding them before they find us, (Princeton: Princeton University Press) 